\title{
Arthroscopic Lunate Excision Provides Excellent Outcomes for Low-Demand Patients with Advanced Kienböck's Disease
}

\author{
Takamasa Shimizu, M.D., Ph.D., Shohei Omokawa, M.D., Ph.D., \\ Kenji Kawamura, M.D., Ph.D., Yasuaki Nakanishi, M.D., Ph.D., \\ Tadanobu Onishi, M.D., Ph.D., Mitsuyuki Nagashima, M.D., Hideo Hasegawa, M.D., Ph.D., \\ Shimpei Kurata, M.D., Ph.D., and Yasuhito Tanaka, M.D., Ph.D.
}

\begin{abstract}
Purpose: To examine the clinical outcomes of arthroscopic lunate excisions for advanced Kienböck's disease. Methods: Fifteen patients (six men and nine women; mean age: 65 years; range: $48-83$ years) with advanced Kienböck's disease, who underwent arthroscopic lunate resection between April 2008 and March 2016, were reviewed clinically and radiographically after a follow-up of $>2$ years (mean: 29 months; range: 24-60 months). Clinical parameters, such as wrist range of motion, grip strength, Disabilities of the Arm, Shoulder, and Hand (DASH) score, and patient-rated wrist evaluation (PRWE) score were evaluated. Radiographic parameters included radioscaphoid angle, scaphocapitate angle, carpal height ratio, ulnar-triquetrum distance, and the scaphoid-triquetrum distance. Wilcoxon's signed-rank test was used to compare measurement results. Results: During the final follow-up, patients exhibited significant improvements, such as $42.9^{\circ}$ in wrist range of motion $(P=.009), 24.5 \%$ of the contralateral side in grip strength $(P=.001), 26.2$ points in DASH score $(P=.002)$, and 37.8 points in PRWE score $(P<.001)$, compared with the preoperative values. The radioscaphoid and scaphocapitate angles significantly increased by $4.8^{\circ}(P=.0027)$ and $3.7^{\circ}$ $(P=.0012)$, respectively. The carpal height ratio, ulnar-triquetrum distance, and scaphoid-triquetrum distance significantly decreased by $0.05(P<.001), 2.6 \mathrm{~mm}(P<.001)$, and $1.3 \mathrm{~mm}(P=.0012)$, respectively. Conclusions: Our results suggest that arthroscopic lunate excisions provided excellent postoperative pain relief and functional recovery within 2 years of follow-up. Changes in carpal alignment and stress concentration on the radial side of the carpal bones could occur in the long term; however, arthroscopic lunate excision can be a good surgical option for treating low-demand patients with advanced Kienböck's disease. Level of Evidence: Level IV, therapeutic case series
\end{abstract}

Department of Orthopedic Surgery, Nara Medical University, Nara, Japan (T.S., Y.N., T.O., M.N., H.H., S.K., Y.T.); Department of Hand Surgery, Nara Medical University, Nara, Japan (S.O.); and Department of Limb Trauma Center, Nara Medical University, Nara, Japan (K.K.)

The authors report the following potential conflicts of interest or sources of funding: This work was supported by JSPS KAKENHI grant number18K16672. Full ICMJE author disclosure forms are available for this article online, as supplementary material.

Received May 30, 2020; accepted June 28, 2021.

Address correspondence to Shohei Omokawa, M.D., Ph.D., Department of Hand Surgery, Nara Medical University, 840 Shijo-cho, Kashihara, Nara, 634-8522, Japan.E-mail: shoheiomokawa@gmail.com

(C) 2021 THE AUTHORS. Published by Elsevier Inc. on behalf of the Arthroscopy Association of North America. This is an open access article under the CC BY-NC-ND license (http://creativecommons.org/licenses/by-nc-nd/4.0/). 2666-061X/20865

https://doi.org/10.1016/j.asmr.2021.06.007

\section{Introduction}

$\mathbf{L}$ unate excision is seldom indicated for the management of Kienböck's disease because of further proximal migration of the capitate and stress concentration on the scaphoid. ${ }^{1}$ Watson et al. postulated that a pattern of carpal collapse and scaphoid rotatory deformity is seen in Kienböck's disease and emphasized that the maintenance of carpal height ratio (CHR) and correction of scaphoid alignment were essential in preventing progressive degenerative change at the radioscaphoid joint. ${ }^{2}$ Lunate excision combined with limited wrist arthrodesis ${ }^{3-5}$ or inserting a spacer $^{6,7}$ is a method of providing biomechanical support to the carpus. However, a literature review suggested that lunate excision's clinical outcomes are comparable to those of other conventional techniques, ${ }^{8}$ and satisfactory results after $>10$ years have been reported. ${ }^{9-11}$ 
The proximal end of the scaphoid is combined with lunate and triquetrum to form a deep concavity that articulates with the convexity of the combined capitate and hamate. After lunate excision, the condyle formed by the capitate and hamate should be supported by newly formed proximal carpal row of the scaphoid and triquetrum. The extrinsic carpal ligaments around the lunate play a crucial role in connecting the scaphoid and triquetrum. Lunate excision has been performed using the dorsal wrist approach, while preserving the volar capsule and a small fragment of the volar lip. ${ }^{10}$ However, one of the reasons for the variations in clinical outcomes and postoperative carpal collapse may be the differences in preserving the dorsal extrinsic carpal ligaments: the radiocarpal (DRC) and dorsal intercarpal (DIC) ligaments. These ligaments are easily damaged in cases of complete lunate excision, as the lunate is attached to the DIC and DRC ligaments in $99 \%$ and $90 \%$ of patients, respectively. ${ }^{12}$ The disruption of these ligaments can cause dysfunction of the proximal carpal row, which leads to proximal migration of the capitate and stress concentration on the scaphoid. The importance of ligament reconstruction has been reported in recent years. ${ }^{7}$

We assumed that the arthroscopic approach could not only remove the lunate from within the wrist joint, but also preserve the dorsal extrinsic carpal ligaments safely. This procedure can prevent the proximal migration of the capitate and stress concentration on the scaphoid without a spacer or limited wrist fusion. However, there is little information available about the clinical outcome after arthroscopic lunate excision for the treatment of Kienböck's disease. The purpose of this study was to examine the clinical outcomes of arthroscopic lunate excision for treating advanced Kienböck's disease. We hypothesized that arthroscopic lunate excision could preserve wrist function and prevent progressive carpal collapse without a spacer.

\section{Methods}

This was a retrospective study performed at our university hospital. Patients with advanced Kienböck's disease were treated between April 2008 and March 2016 with arthroscopic lunate excision and reviewed clinically and radiographically after a follow-up of $>2$ years was included. This procedure was strongly recommended for unreconstructable lunates in patients aged $>65$ years, regardless of their physical activity level, and in low-demand patients aged $<65$ years. Patients aged $<65$ years who were manual workers and participated in high-demand sports activities were indicated for a vascularized bone graft, vascularized osteochondral bone graft from the femur, ${ }^{13}$ or radiolunate fusion with a vascularized bone graft. ${ }^{14}$ Patients who were not reviewed, both, clinically and radiographically, and those who participated in follow- up for less than 2 years, were excluded from the study. The study received Institutional Review Board approval from Nara Medical University (study no. 2547).

Unreconstructable lunates were defined as a highly fragmented lunate and its shell structure largely collapsed with joint incongruence in the radiolunate or midcarpal joint on computed tomography (CT) scans. Unreconstructable lunate evaluations were also based on other imaging evaluations, arthroscopic findings, age, and physical activity level. Imaging findings included radiography and magnetic resonance imaging (MRI). Partial or total necrosis was diagnosed using T2weighted MRI. Regarding arthroscopic findings, the articular surface was evaluated on the basis of the Bain and Begg arthroscopic classification. ${ }^{4}$ A nonfunctional articular surface has at least one of the following: extensive fibrillation, fissuring, localized or extensive loss, a floating articular surface, or fracture. Bain grades $2 \mathrm{~b}, 3$, and 4 were defined as unreconstructable lunates.

\section{Surgical Approach}

A traction tower was applied with 10-15 lbs of traction. First, a $1.9-\mathrm{mm}, 30^{\circ}$ oblique arthroscope was inserted through the 3-4 portal, and the articular cartilage of the radiocarpal joint was viewed. Surgical instruments were inserted through the 4-5 portal, and a synovectomy was performed. The degeneration or tear of the scapholunate (SL) ligament, lunotriquetral (LT) ligament, or the triangular fibrocartilage complex were examined with a surgical probe. Even if there is degeneration of the SL and LT ligaments, reconstruction is unnecessary because the lunate is resected; if there is degeneration of the triangular fibrocartilage complex, debridement of the degenerated portion can be considered along with synovectomy. Next, the arthroscope was inserted through the radial midcarpal portal, and surgical instruments were inserted through the ulnar midcarpal portal to evaluate the articular surface of the midcarpal joint (Fig 1). On the basis of the Bain and Begg arthroscopic classification, ${ }^{15}$ extensive fibrillation, fissuring, localized or extensive loss, a floating articular surface, or fracture were nonfunctional articular surfaces.

Before lunate excision, it was recommended that the membranous portion of the SL and LT ligaments be cut with a radiofrequency probe to avoid accidental resection of the scaphoid and triquetrum. In cases with SL and LT ligamentous tear or a complicated degree of fragmentation, the initial marking of these ligaments' location is important because the ligament may be misplaced in inadequate lunate excision. First, the lunate's proximal part on the dorsal aspect was resected through the radiocarpal joints with the ablation bar (Fig 2A). Next, the volar segment of the lunate was resected through the midcarpal portal (Fig 2B). Finally, 


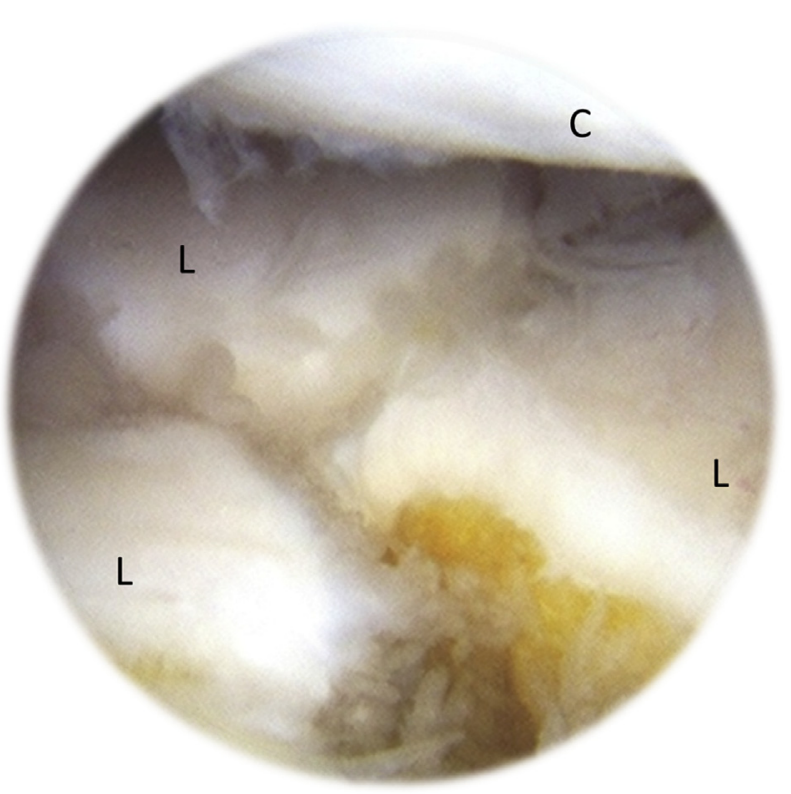

Fig 1. Arthroscopic findings of the lunate. Arthroscopic findings of the right wrist $\left(30^{\circ}\right.$ scope). View from the radial midcarpal portal showing that the articular surface of the lunate in the midcarpal joint was severely fragmented and unreconstructable. C, capitate head; L, fragmented lunate.

the arthroscope was inserted through the volar portal. The remaining distal part of the lunate on the dorsal aspect was resected with the ablation bar inserted through the 3-4 portal or 4-5 portal (Fig 2C). The dorsal and volar SL and LT ligaments and attachment sites of each ligament were preserved. Other extrinsic ligaments that attach to the volar and dorsal cortexes of the lunate were also preserved.

During the postoperative course, a short-arm cast was applied from the immediate postoperative period until 3 weeks postoperatively. Physiotherapy of the wrist joint was started after the cast was removed. A wrist immobilization orthosis was applied from 3 weeks to 3 months postoperatively when using the affected limbs.

\section{Clinical Evaluation}

An orthopedic and trauma surgeon (K.K.), unaware of the patient medical history, completed the following measurements at the final follow-up. Clinical outcome evaluations included the wrist range of motion (ROM) (flexion-extension) and grip strength (\% relative to the contralateral side). The ROM of the wrist was assessed with a handled goniometer, and the grip strength was measured 3 times, with a 30-second interval between measurements, using a Jamar dynamometer (Jamar; Smith and Nephew, Memphis, TN).

Patients were asked to respond to the Disabilities of the Arm, Shoulder, and Hand (DASH) and PatientRated Wrist Evaluation (PRWE) questionnaires. The DASH is a 30 -item self-report questionnaire that measures physical function and symptoms in patients with musculoskeletal disorders of the upper limb. ${ }^{16}$ The PRWE is a validated 15-item wrist-specific questionnaire for pain and function and has been used to assess
Fig 2. Schema of the lunate resection (lateral view). (A) Resection of the dorsoproximal part of the lunate from the radiocarpal joint (1). (B) Resection of the volar part of the lunate from the midcarpal joint (2). (C) Resection of the dorsal distal part through the volar portal (3). $\mathrm{C}$ : capitate; $\mathrm{R}$, radius.

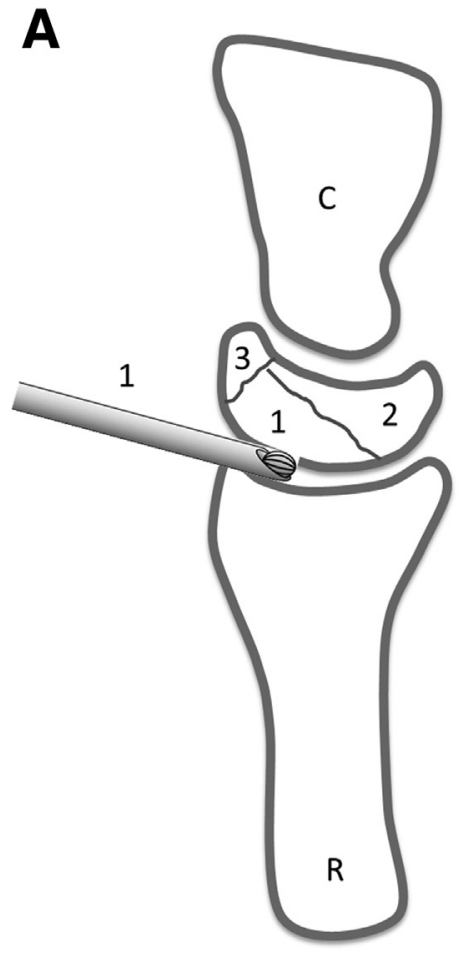

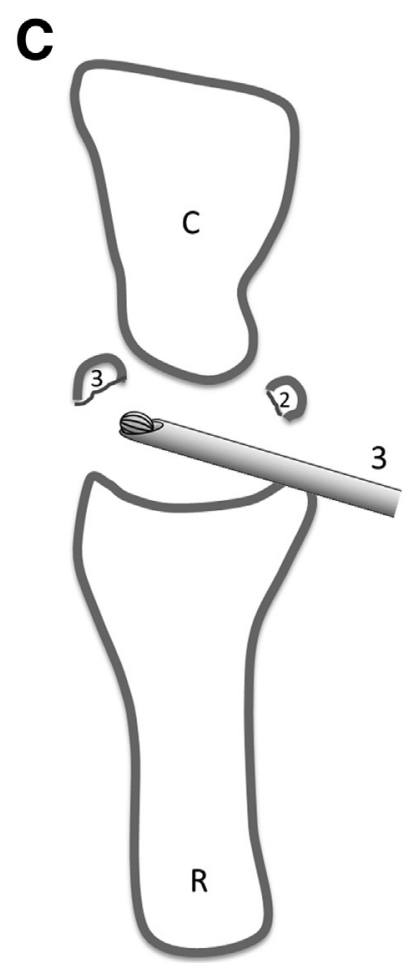



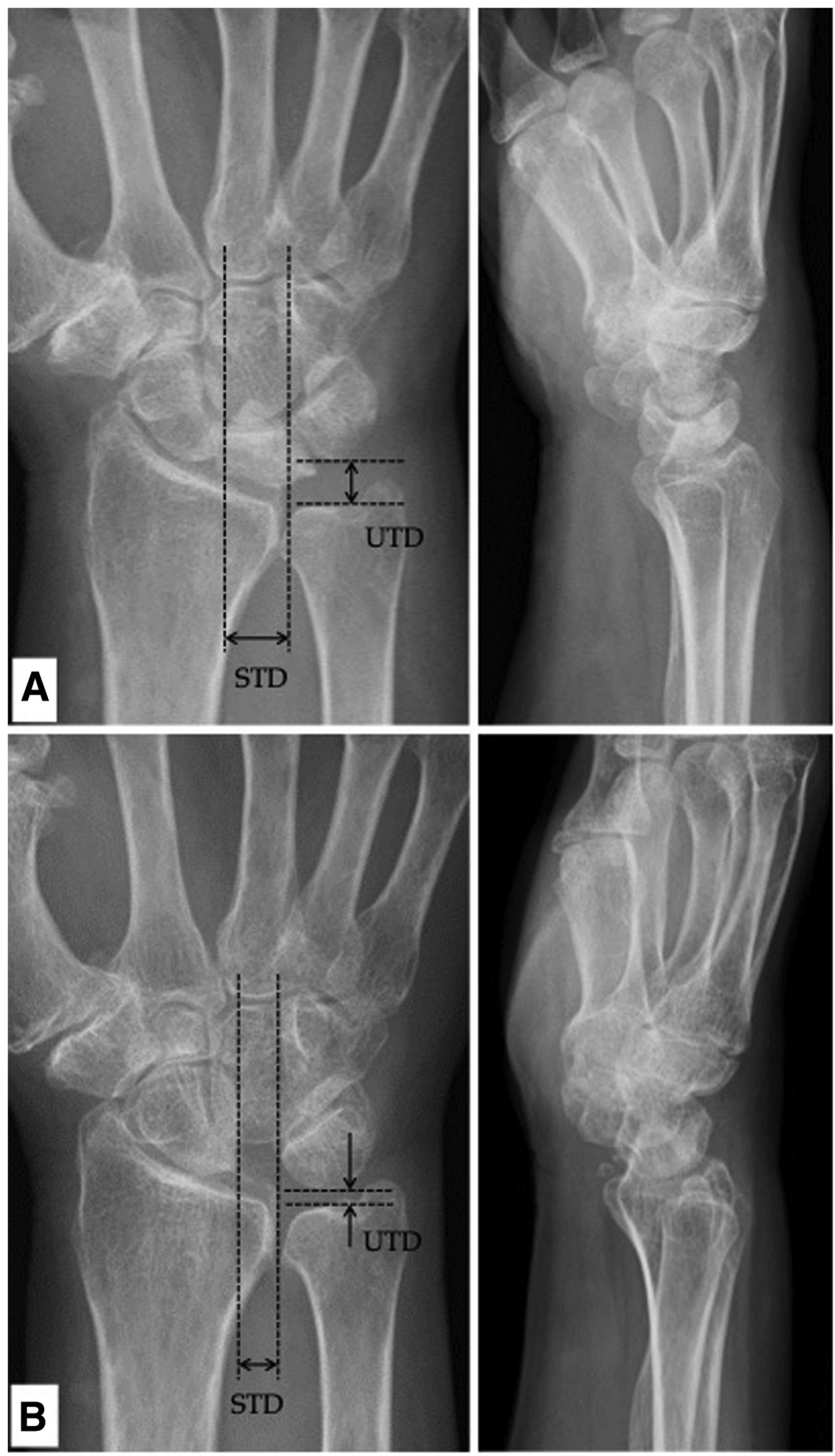

Fig 3. Preoperative and postoperative radiographs of standard anterior-posterior and lateral view of the right wrist (A and B). Ulnar-triquetrum distance (UTD) was measured as the distance between the ulnar head and the proximal ridge of the triquetrum. Scaphoid-triquetrum distance (STD) was measured as the distance between the ulnar ridge of the scaphoid and the radial ridge of the triquetrum. subjective outcomes in patients with wrist injuries. ${ }^{17,18}$ Both questionnaires are scored from 0 to 100, with lower scores indicating a better condition.

Statistically clinically significant outcome improvement in patient-rated outcomes was determined using the minimal clinically important difference (MCID), as previously established in the literature. ${ }^{19,20}$ MCID was defined as the minimal difference in a patient-rated outcome perceived by the patient as being beneficial because there are small differences that patients cannot perceive, even if they are statistically significant. During this study, MCID was defined as more than a 10-point 
Table 1. Preoperative and Postoperative Clinical Results

\begin{tabular}{lcrr}
\hline & Postoperative & Difference in Postoperative \\
to Preoperative & (Final Follow-Up) & $42.9 \pm 27.4$ \\
Flexion-extension (degree) & $77.5 \pm 32.7$ & $120.4 \pm 25.9$ & $24.5 \pm 29.4$ \\
Grip strength (\% vs contralateral) & $54.6 \pm 22.4$ & $77.1 \pm 14.4$ & -009 \\
DASH score & $38.0 \pm 24.7$ & $13.4 \pm 14.1$ & .001 \\
PRWE & $49.6 \pm 23.8$ & $13.2 \pm 14.5$ & $-37.8 \pm 23.2$ \\
\hline
\end{tabular}

DASH, Disabilities of the Arm, Shoulder, and Hand; PRWE, patient-rated wrist evaluation.

change in the DASH scores and more than a 14-point change in the PRWE scores. ${ }^{21}$ In addition, because a wrist with a DASH and PRWE score of 20 points or less has been considered free of disability, we defined an excellent outcome as a final DASH and PRWE score of 20 points or less. $^{22}$

\section{Radiographic Evaluation}

Posteroanterior and lateral views of the preoperative and postoperative wrist radiographs were acquired with the forearm in the neutral rotation. All the preoperative and postoperative evaluations were performed by two orthopedic surgeons (M.N. and H.H.). The radiographic parameter, including the $\mathrm{CHR}$, radioscaphoid angle (RSA), scaphocapitate angle (SCA), and ulnar variance (UV) were measured. The ulnar-triquetrum distance (UTD) was measured as the distance between the ulnar head and the proximal ridge of the triquetrum (Fig 3). The scaphoid-triquetrum distance (STD) was measured as the distance between the ulnar ridge of the scaphoid and the radial corner of the triquetrum (Fig 3). The radiographs were randomly distributed to two examiners after removing any identifying patient's information. To avoid recall bias, the examiners repeated their measurements on all radiographs in a similar manner following a period of at least 2 weeks from the initial assessment.

\section{Statistical Analysis}

Wilcoxon's signed-rank test was used to determine differences between preoperative and postoperative variables of the ROM, grip strength, DASH scores, PRWE scores, and radiologic parameters. A $P$ value $<.05$ was considered statistically significant. The radiographic parameters were continuous variables, and interclass correlation coefficients were used to assess inter-rater and intrarater reliabilities. Data analysis was conducted using the Statistical Package for the Social Sciences (SPSS) software package (version 22.0; SPSS Inc., Chicago, IL).

\section{Results}

We identified 17 consecutive patients treated from April 2008 and March 2016 eligible for this study, of which 2 patients were excluded because of the short follow-up period. Thus, 15 wrists (patients) were included in the study cohort.
Fifteen patients (six men, nine women; mean age: 65 years; range: $48-83$ years) were reviewed during this study. The mean follow-up period was 29 months (range: 24-60 months). Using the radiographic staging, according to the Lichtman classification, one patient was classified as stage IIIB, 13 patients were classified as stage IIIC, and one patient was classified as stage IV. During the intraoperative arthroscopic evaluation of the articular cartilage, according to the Bain and Begg arthroscopic classification, ${ }^{4} 11$ patients were classified as grade $2 \mathrm{~b}, 3$ patients were classified as grade 3 , and 1 patient was classified as grade 4 .

During the final follow-up, the patients exhibited significant improvements, such as $42.9^{\circ}$ in wrist ROM $(P=.009), 24.5 \%$ of the contralateral side in grip strength $(P=.001), 26.2$ points in DASH score $(P=.002)$, and 37.8 points in PRWE score $(P<.001)$ compared with the preoperative values (Table 1). After surgery, 11 of the 15 patients $(73.3 \%)$ showed clinically significant improvement in the DASH score, and 13 of the 15 patients $(86.7 \%)$ showed clinically significant improvement in the PRWE score. An excellent outcome was achieved in 13 of 15 patients $(86.7 \%)$ in the DASH score and 12 of 15 patients $(80.0 \%)$ in the PRWE score.

Regarding the radiographic evaluation, CHR, RSA, SCA, UV, UTD, and STD showed excellent intra-rater reliability (0.77-0.99). CHR, RSA, and SCA showed good and fair inter-rater reliability (0.52-0.73). UV, UTD, and STD showed excellent inter-rater reliability (0.91-0.97). After arthroscopic lunate excision, CHR significantly decreased by an average of 0.05. RSA and SCA significantly increased by an average of $4.8^{\circ}$ and $3.7^{\circ}$, respectively. UV did not significantly change. UTD and STD significantly decreased by $2.6 \mathrm{~mm}$ and 1.3 $\mathrm{mm}$, respectively (Table 2). There is no osteoarthritic change in the radiocarpal and midcarpal joints.

\section{Complications}

Four patients with positive ulnar variance $>2 \mathrm{~mm}$ had postoperative sclerotic changes in the ulnocarpal joint, but none reported severe joint pain.

\section{Discussion}

Our investigation demonstrated that arthroscopic lunate excision could provide adequate pain relief and optimal patient-rated functional outcomes and improve 
Table 2. Preoperative and Postoperative Radiological Results

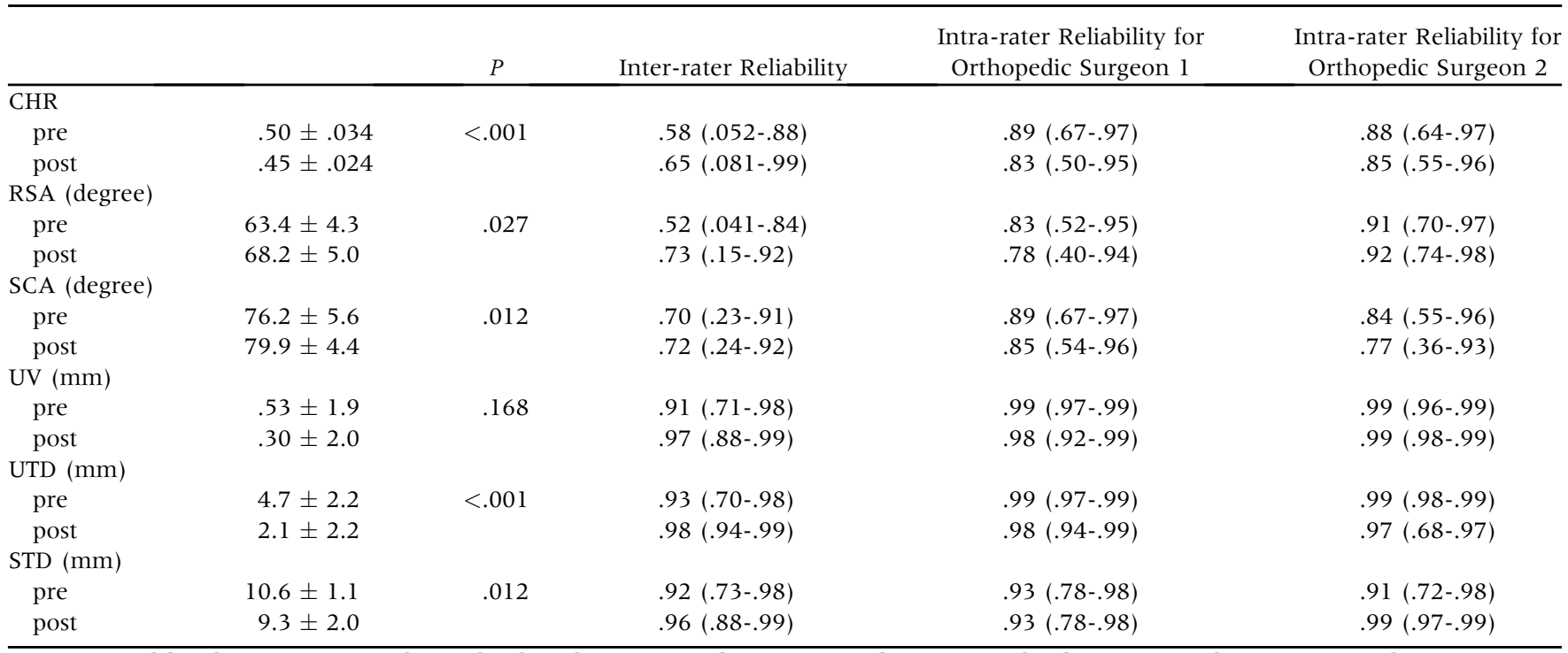

CHR, carpal height ratio; RSA, radioscaphoid angle; SCA, scaphocapitate angle; STD, scaphoid-triquetrum distance; UTD, ulnar-triquetrum distance; UV, ulnar variance.

objective findings of wrist ROM and grip strength, with minimal disability within 2 years of follow-up. Furthermore, none of the patients underwent additional surgery during the 2-year follow-up. The patient-rated outcome evaluation, including DASH score and PRWE, demonstrated that more than $73 \%$ of patients showed clinically significant improvement, and more than $80 \%$ of patients showed an excellent outcome. This procedure has several advantages. First, it can preserve the range of wrist motion in both the radiocarpal and midcarpal joints. The limited wrist fusion with lunate excisions could provide the postoperative wrist range of motion (flexion-extension) ranged from $66^{\circ}$ to $85^{\circ}$ in long-term follow-up..$^{3-5}$ However, arthroscopic lunate excision could provide averaged $120^{\circ}$ in the 2 -year follow-up. Second, it needs only short-term postoperative therapy. Although limited wrist fusions require wrist immobilization for 6 to 12 weeks with short or long arm casts, ${ }^{3-5}$ arthroscopic lunate excision requires 3 weeks of immobilization with a short arm cast, which is similar to the postoperative course of proximal row carpectomy. ${ }^{23,24}$ The current study demonstrated that the arthroscopic lunate excision has the potential to become a widely used surgical option for the low-demand patients of advanced Kienböck's disease with unreconstructable lunates.

Progressive carpal collapse, including scaphoid volar flexion, decreased carpal height, and ulnar translation were inevitable complications after lunate excision. Contrastingly, Rhee et al. reported that the scaphocapitate arthrodesis with lunate excision in advanced Kienböck's disease significantly decreased the mean MCHR; however, symptomatic carpal collapse was not evident until a mean of 5 years of follow-up. ${ }^{4}$ Charre et al. also reported that the scaphocapitate arthrodesis with lunate excision demonstrated that the degree of correction of scaphoid flexion does not significantly affect clinical outcome, and there were only two cases $(11.7 \%)$ of styloscaphoid arthritis in the mean 10.7 years of follow-up. ${ }^{3}$ These reports suggested stabilizing the midcarpal joint acts protectively against the progressive carpal collapse in the wrist joint. During this study, with the minimum of a 2 -year follow-up period, the CHR decreased. The scaphoid flexed in the volar direction with an average of $4.8^{\circ}$ in RSA and $3.7^{\circ}$ in SCA; however, the STD decreased with an average of $1.3 \mathrm{~mm}$ postoperatively. By sandwiching the condyle formed by the capitate and hamate between the scaphoid and the triquetrum, the load can be distributed to the scaphoid and triquetrum. None of the patients developed degenerative changes at the radioscaphoid joint. We assum that the extrinsic carpal ligament can provide stability for the midcarpal joint and have a protective effect on the progressive carpal collapse in the wrist joint. However, unlike arthrodesis, there is a possibility that the reserved ligaments may degenerate with long-term loading. It is necessary to pay attention to the appearance of the degenerative changes in the long-term follow-up.

Menth-Chiari et al. ${ }^{25}$ first reported the outcomes of arthroscopic debridement; all seven patients in that study with advanced Kienböck's disease showed significant improvements in pain symptoms and experienced complete relief of mechanical symptoms. However, in their study, 3 patients were followed up for 6 months, and 2 of the remaining 4 patients who participated in long-term follow-up (18-42 months) had radiographic images showing that the remaining 
lunate had developed degenerative changes. Five of those 7 patients had a complete SL ligament tear or LT ligament tear, which caused further fragmentation of the remaining lunate and carpal instability, similar to the scapholunate advanced collapse wrist. Watson et al. demonstrated that $32 \%$ of STT fusion for Kienböck's disease required lunate excision for additional surgery. ${ }^{2}$ To avoid degeneration of the central column at the radiolunate and midcarpal articulations, complete arthroscopic lunate excision was preferred over arthroscopic lunate debridement for patients with unreconstructable lunates. Concerning the appropriate amount of the lunate excision, we believe it is appropriate to remove all but the dorsal and volar cortex of the lunate. Future biomechanical studies will have to confirm the effects of maintaining these carpal ligaments and the wrist joint's associated kinematics.

In the current study, the UTD decreased by approximately $2.6 \mathrm{~mm}$ from $4.7 \mathrm{~mm}$ before surgery to $2.1 \mathrm{~mm}$ after surgery. Three patients with preoperative UTD less than $3 \mathrm{~mm}$ developed sclerotic changes in the ulnocarpal joint after lunate excision. Kawai et al. also reported that the ulnotriquetral distance decreases 2.2 $\mathrm{mm}$ (on an average) after lunate excision. ${ }^{10}$ These results suggested that patients with preoperative UTD less than $3 \mathrm{~mm}$ are at a risk of developing the ulno-carpal impaction syndrome in the long term. Kienböck's disease in elderly patients exhibits different behavior than in pediatric and adult cohorts, ${ }^{26}$ and the majority of patients with Kienböck's disease older than 70 years were associated with positive $\mathrm{UV} .{ }^{27}$ In the current study, the average age was 65 years, and 14 of 15 patients (93.3\%) had positive UV. Because Kienböck's disease in elderly patients tends with positive UV and smaller UTD, the surgeons should be aware of the risk of postoperative ulno-carpal impaction syndrome and should explain the possibility of additional arthroscopic surgery, such as wafer procedure ${ }^{28}$ in the application of arthroscopic lunate excision.

\section{Limitations}

The main limitations of this study were the use of a retrospective case series design and a short-term followup period. Additionally, the number of patients was small; there was no comparative treatment due to lack of a control group. Furthermore, it is necessary to examine the changes in pressure distribution in the proximal carpal row after arthroscopic lunate excision in the biomechanical study. This study aimed to look at a small cohort of low-demand patients, and these outcomes may not be generalized to younger, higher-demand patients.

\section{Conclusion}

Our results suggested that arthroscopic lunate excisions provided excellent postoperative pain relief and functional recovery within 2 years of follow-up. Changes in carpal alignment and stress concentration on the radial side of the carpal bones could occur in the long term; however, arthroscopic lunate excision can be a good surgical option for treating low-demand patients with advanced Kienböck's disease.

\section{Acknowledgment}

The authors thank Hiroshi Ono M.D., Ph.D., Tsutomu Kira, M.D. Ph.D., and Naoki Hayami, M.D. Ph.D. for their valuable help in this study. We would like to thank Editage (www.editage.jp) for English language editing.

\section{References}

1. Lichtman DM, Pientka WF 2nd, Bain GI. Kienböck disease: A new algorithm for the 21st century. J Wrist Surg 2017;6:2-10.

2. Watson HK, Monacelli DM, Milford RS, Ashmead DI. Treatment of Kienböck's disease with scaphotrapeziotrapezoid arthrodesis. J Hand Surg Am 1996;21:9e15.

3. Charre A, Delclaux S, Apredoai C, Ayel JE, Rongieres M, Mansat P. Results of scaphocapitate arthrodesis with lunate excision in advanced Kienböck disease at 10.7-year mean follow-up. J Hand Surg Eur 2018;43:362-368.

4. Rhee PC, Lin IC, Moran SL, Bishop AT, Shin AY. Scaphocapitate arthrodesis for Kienböck disease. J Hand Surg Am 2015:40:745-751.

5. Lee JS, Park MJ, Kang HJ. Scaphotrapeziotrapezoid arthrodesis and lunate excision for advanced Kienböck disease. J Hand Surg Am 2012;37:2226-2232.

6. Viljakka T, Tallroth K, Vastamäki M. Long-term clinical outcome after titanium lunate arthroplasty for Kienböck disease. J Hand Surg Am 2018;43:945.el-945.e10.

7. Aslantürk O, Ertem K, Ergen E, Köroğlu M. Extensor carpi radialis longus tendon ball interposition arthroplasty for treatment of late-stage Kienböck's disease: Short-term results of a novel technique. J Orthop Surg (Hong Kong) 2018;26:2309499018802514.

8. Wheatley MJ, Finical SJ. A 32-year follow-up of lunate excision for Kienbock's disease: A case report and a review of results from excision and other treatment methods. Ann Plast Surg 1996;37:322-325.

9. Blanco RH. Excision of the lunate in Kienböck's disease: Long-term results. J Hand Surg Am 1985;10:1008-1013.

10. Kawai H, Yamamoto K, Yamamoto T, Tada K, Kaga K. Excision of the lunate in Kienböck's disease. Results after long-term follow-up. J Bone Joint Surg Br 1988;70: 287-292.

11. Blanco RH, Blanco FR, Cervigni F. Kienböck Disease: Postoperative radiographs at long-term follow-up. J Wrist Surg 2016;5:110-112.

12. Mitsuyasu H, Patterson RM, Shah MA, Buford WL, Iwamoto Y, Viegas SF. The role of the dorsal intercarpal ligament in dynamic and static scapholunate instability. J Hand Surg Am 2004;29:279-288.

13. Pet MA, Assi PE, Giladi AM, Higgins JP. Preliminary clinical, radiographic, and patient-reported outcomes of 
the medial femoral trochlea osteochondral free flap for lunate reconstruction in advanced Kienböck disease. J Hand Surg Am 2020;45:774.el-774.e8.

14. Ono H, Furuta K, Fujitani R. Radiolunate fusion using vascularized radius bridging graft for the treatment of advanced Kienböck disease: two cases report. Hand Surg 2010;15:139-144.

15. Bain GI, Begg M. Arthroscopic assessment and classification of Kienbock's disease. Tech Hand Up Extrem Surg 2006;10:8-13.

16. Changulani M, Okonkwo U, Keswani T, Kalairajah Y. Outcome evaluation measures for wrist and hand. Which one to choose? Int Orthop 2008;32:1-6.

17. MacDermid JC. Development of a scale for patient rating of wrist pain and disability. J Hand Ther 1996;9: 178-183.

18. MacDermid JC, Richards RS, Donner A, Bellamy N, Roth JH. Responsiveness of the short form-36, disability of the arm, shoulder, and hand questionnaire, patientrated wrist evaluation, and physical impairment measurements in evaluating recovery after a distal radius fracture. J Hand Surg Am 2000;25:330-340.

19. Harris JD, Brand JC, Cote MP, Faucett SC, Dhawan A. Research pearls: The significance of statistics and perils of pooling. Part 1: Clinical versus statistical significance. Arthroscopy 2017;33:1102-1112.

20. Chan LS. Minimal clinically important difference (MCID). Adding meaning to statistical inference. Am J Public Health 2013;103:e24-e25.
21. Sorensen AA, Howard D, Tan WH, Ketchersid J, Calfee RP. Minimal clinically important differences of 3 patient-rated outcomes instruments. J Hand Surg Am 2013;38:641-649.

22. Grewal R, MacDermid JC. The risk of adverse outcomes in extra-articular distal radius fractures is increased with malalignment in patients of all ages but mitigated in older patients. J Hand Surg 2007;32:962-970.

23. Lumsden BC, Stone A, Engber WD. Treatment of advanced-stage Kienböck's disease with proximal row carpectomy: an average 15-year follow-up. J Hand Surg Am 2008;33:493-502.

24. Giacalone F, di Summa PG, Fenoglio A, et al. Resurfacing capitate pyrocarbon implant versus proximal row carpectomy alone: A comparative study to evaluate the role of capitate prosthetic resurfacing in advanced carpal collapse. Plast Reconstr Surg 2017;140:962-970.

25. Menth-Chiari WA, Poehling GG, Wiesler ER, Ruch DS. Arthroscopic debridement for the treatment of Kienbock's disease. Arthroscopy 1999;15:12-19.

26. Farr S, Ganger R, Girsch W. Vascularized bone transfer for the treatment of advanced-stage juvenile lunatomalacia: A report of two cases. J Pediatr Orthop B 2014;23:282-284.

27. Taniguchi Y, Yoshida M, Iwasaki H, Otakara H, Iwata S. Kienböck's disease in elderly patients. J Hand Surg Am 2003;28:779-783.

28. Oh WT, Kang HJ, Chun YM, Koh IH, An HM, Choi YR. Arthroscopic wafer procedure versus ulnar shortening osteotomy as a surgical treatment for idiopathic ulnar impaction syndrome. Arthroscopy 2018;34:421-430. 